\title{
ON THE ISOPERIMETRIC CONSTANT OF SYMMETRIC SPACES OF NONCOMPACT TYPE
}

\author{
XIAODONG WANG \\ (Communicated by Guofang Wei)
}

\begin{abstract}
We prove that the isoperimetric constant is positive for all symmetric spaces of noncompact type and compute it explicitly.
\end{abstract}

Let $N^{n}$ be a complete, noncompact Riemannian manifold. We consider the isoperimetric constant $I(N)$ defined by

$$
I(N)=\inf _{\Omega} \frac{A(\partial \Omega)}{V(\Omega)}
$$

where $\Omega$ ranges over open submanifolds of $N$ with compact closure and smooth boundary. This is also called Cheeger's constant [Ch. The importance of this global geometric invariant is illustrated by the following fundamental inequality relating it to another global analytic invariant:

$$
\lambda_{0}(N) \geq I(N)^{2} / 4
$$

where $\lambda_{0}$ is the bottom of the spectrum of the Laplace operator on $N$. It is a well-known fact that $\lambda_{0}$ can be characterized as

$$
\lambda_{0}=\inf \frac{\int_{N}|\nabla f|^{2}}{\int_{N}|f|^{2}},
$$

where $f$ ranges over nonzero $C^{1}$ functions with compact support.

For $\mathbb{R}^{n}$ or any Riemannian manifold with nonnegative Ricci curvature, the isoperimetric constant $I$ is zero. On the other hand, for Cartan-Hadamard manifolds with sectional curvature bounded by a negative constant from above, Yau proved that $I$ is always positive.

Proposition 1 (Yau $[\mathrm{Y}]$ ). If $N$ is simply connected with sectional curvatures $\leq$ $\kappa<0$, then

$$
I(N) \geq(n-1) \sqrt{-k} .
$$

From this result one can easily deduce $I\left(\mathbb{H}^{n}\right)=n-1$. For a detailed discussion of Cheeger's constant and related results, one can consult [Cha, Chapter 6]. In general, it is very difficult to know if the isoperimetric constant is positive or not and it is almost impossible to compute it explicitly if it is known to be positive. In this short note, we prove that the isoperimetric constant is positive for all symmetric spaces of noncompact type and compute it explicitly (see Theorem 1).

Received by the editors June 30, 2014 and, in revised form, July 29, 2014.

2010 Mathematics Subject Classification. Primary 53C35; Secondary 58J50. 
Let $\left(\widetilde{M}^{n}, g\right)$ be a Cartan-Hadamard manifold (i.e. complete, simply-connected with nonpositive curvature) and $\mathcal{S} \widetilde{M}$ its unit tangent bundle. For any $p \in M$ and $u \in \mathcal{S}_{p} \widetilde{M}$ we have a nonnegative symmetric operator $R_{u}: T_{p} \widetilde{M} \rightarrow T_{p} \widetilde{M}$ defined by

$$
R_{u}(X)=-R(u, X) u .
$$

Let $0=\lambda_{0}(u) \leq \lambda_{1}(u) \leq \cdots \leq \lambda_{n-1}(u)$ be its eigenvalues. In this way we have $n$ continuous functions $\lambda_{0}, \cdots, \lambda_{n-1}$ on $\mathcal{S} \widetilde{M}$. Obviously $\lambda_{i}(-u)=\lambda_{i}(u)$.

From now on we assume $\widetilde{M}$ is a symmetric space of noncompact type. By this we mean that $\widetilde{M}$ is a Cartan-Hadamard manifold with parallel curvature tensor and there is no Euclidean factor in its de Rham decomposition. When $\widetilde{M}$ has rank one, it is negatively curved. But if the rank is higher, its sectional curvature vanishes on certain 2-planes. A standard reference on symmetric spaces is Helgason $[\mathrm{H}]$. Another excellent sourse is the book $[\mathrm{E}$ by Eberlein.

We fix a base point $o \in \widetilde{M}$. For $\xi \in \mathcal{S}_{o} \widetilde{M}$ let $\gamma_{\xi}$ be the geodesic ray with initial velocity $\xi$. We can choose an orthonormal basis $\left\{e_{1}, \cdots, e_{n-1}\right\}$ for $\xi^{\perp}$ s.t.

$$
R_{\xi} e_{i}=-R\left(\xi, e_{i}\right) \xi=\lambda_{i}(\xi) e_{i} .
$$

Let $E_{i}$ be the parallel vector field along $\gamma_{\xi}$ with $E_{i}(0)=e_{i}$. Since the curvature tensor is parallel, we have along $\gamma_{\xi}$

$$
R_{\gamma_{\xi}^{\prime}(t)} E_{i}(t)=-R\left(\gamma_{\xi}^{\prime}(t), E_{i}(t)\right) \gamma_{\xi}^{\prime}(t)=\lambda_{i}(\xi) E_{i}(t) .
$$

Therefore

$$
\lambda_{i}\left(\gamma_{\xi}^{\prime}(t)\right)=\lambda_{i}(\xi)
$$

This proves that the $n$ continuous functions $\lambda_{0}, \cdots, \lambda_{n-1}$ on $\mathcal{S} \widetilde{M}$ are invariant under the geodesic flow.

Along the geodesic $\gamma=\gamma_{\xi}$ the Jacobi field equation

$$
X^{\prime \prime}(t)+R\left(\gamma^{\prime}, X\right) \gamma^{\prime}=0
$$

can be explicitly solved. The solution satisfying the initial condition $X(0)=$ $0, X^{\prime}(0)=e_{i}$ is given by

$$
X_{i}(t)=\frac{\sinh \sqrt{\lambda_{i}(\xi)} t}{\sqrt{\lambda_{i}(\xi)}} E_{i}(t) .
$$

For any integer $k \geq 1$, define the function $b_{k}$ on $\widetilde{M}$ by $b_{k}(x)=d\left(x, p_{k}\right)-k$, where $p_{k}=\gamma_{\xi}(k)$. The Busemann function $b_{\xi}$ is the limit of $b_{k}$ as $k \rightarrow \infty$, i.e.,

$$
b_{\xi}(x)=\lim _{k \rightarrow \infty} d\left(x, p_{k}\right)-k .
$$

It is well known that the limit exists. By [HI], the convergence is locally uniform in $C^{2}(\widetilde{M})$ and in particular $b_{\xi} \in C^{2}(\widetilde{M})$.

Lemma 1. $\Delta b_{\xi}$ is constant and equals $l(\xi):=\sum_{i} \sqrt{\lambda_{i}(\xi)}$.

Proof. We fix $x$ and denote $l_{k}=d\left(x, p_{k}\right)$. Let $\sigma_{k}:\left[0, l_{k}\right] \rightarrow M$ be the geodesic from $x$ to $p_{k}$. We write $u_{k}=-\gamma_{\xi}^{\prime}(k), v_{k}=-\sigma^{\prime}\left(l_{k}\right)$. We have

$$
\Delta b_{k}(x)=\sum_{i} \sqrt{\lambda_{i}\left(v_{k}\right)} \operatorname{coth} \sqrt{\lambda_{i}\left(v_{k}\right)}\left(b_{k}(x)+k\right) .
$$


Let $\theta_{k}=\angle\left(u_{k}, v_{k}\right)$ be the angle between $u_{k}$ and $v_{k}$. By the cosine law,

$$
\cos \theta_{k} \geq \frac{k^{2}+l_{k}^{2}-d(o, x)^{2}}{2 k l_{k}} .
$$

As $\left|l_{k}-k\right| \leq d(o, x)$, it is obvious that $\theta_{k} \rightarrow 0$ as $k \rightarrow \infty$. For each $k$, there exists $\phi_{k} \in G$ s.t. $\phi_{k}\left(p_{k}\right)=o$. Let $\widetilde{u}_{k}=\phi_{*}\left(u_{k}\right), \widetilde{v}_{k}=\phi_{*}\left(v_{k}\right)$. They are unit vectors at $o$ and

$$
\angle\left(\widetilde{u}_{k}, \widetilde{v}_{k}\right)=\angle\left(u_{k}, v_{k}\right) \rightarrow 0
$$

as $k \rightarrow \infty$. By continuity, for each $i$ we have

$$
\left|\lambda_{i}\left(\widetilde{u}_{k}\right)-\lambda_{i}\left(\widetilde{v}_{k}\right)\right| \rightarrow 0
$$

as $k \rightarrow \infty$. Since $\lambda_{i}\left(\widetilde{u}_{k}\right)=\lambda_{i}\left(u_{k}\right)=\lambda_{i}(\xi)$ and $\lambda_{i}\left(\widetilde{v}_{k}\right)=\lambda_{i}\left(v_{k}\right)$, we have $\lambda_{i}\left(v_{k}\right) \rightarrow$ $\lambda_{i}(\xi)$. As $b_{k} \rightarrow b_{\xi}$ in $C_{l o c}^{2}$, we obtain from (0.4) by taking limit

$$
\Delta b_{\xi}(x)=\sum_{i} \sqrt{\lambda_{i}(\xi)}
$$

Let $G=I_{0}(\widetilde{M})$ be the connected component of the isometry group containing the identity and $K=\{\phi \in G: \phi o=o\}$. Thus $G$ is a semisimple Lie group acting transitively on $\widetilde{M}$ by isometries and $K$ a maximal compact subgroup of $G$. Define

$$
\begin{aligned}
\mathfrak{g} & =\{\text { Killing vector fields on } M\}, \\
\mathfrak{t} & =\{X \in \mathfrak{g}: X(o)=0\}, \\
\mathfrak{p} & =\{X \in \mathfrak{g}: \nabla X(o)=0\} .
\end{aligned}
$$

We know that $\mathfrak{g}$ is the Lie algebra of $G$ and $\mathfrak{t}$ is the Lie algebra of $K$. Moreover

$$
\mathfrak{g}=\mathfrak{t} \oplus \mathfrak{p}
$$

and $\mathfrak{p}$ is naturally identified with $T_{o} \widetilde{M}$. Let $\sigma: \mathfrak{g} \rightarrow \mathfrak{g}$ be the Cartan involution, i.e., it is the automorphism s.t. $\left.\sigma\right|_{\mathfrak{t}}=I,\left.\sigma\right|_{\mathfrak{p}}=-I$. Let $B$ be the Killing form $\mathfrak{g}$, i.e., for $X, Y \in \mathfrak{g}$

$$
B(X, Y)=\operatorname{tr}\left(\operatorname{ad}_{X} \operatorname{ad}_{Y}\right) .
$$

Since $\operatorname{ad}_{\sigma(X)}=\sigma \circ \operatorname{ad}_{X} \circ \sigma^{-1}, B$ is invariant under $\sigma$. In particular $\mathfrak{t}$ and $\mathfrak{p}$ are orthogonal to each other w.r.t. the Killing form of $\mathfrak{g}$, i.e., $B(X, Y)=0$ for any $X \in \mathfrak{t}, Y \in \mathfrak{p}$. Moreover, $B$ is negative definite on $\mathfrak{t}$ and positive definite on $\mathfrak{p}$. Thus

$$
\langle X, Y\rangle=-B(\sigma X, Y)
$$

is a metric on $\mathfrak{g}$. To fix the scale, we assume that the Riemannian metric on $T_{o} \widetilde{M}=\mathfrak{p}$ coincides with the restriction of $\langle\cdot, \cdot\rangle$ on $\mathfrak{p}$.

Let $\mathfrak{a} \subset \mathfrak{p}$ be a maximal abelian subspace. For any $\alpha \in \mathfrak{a}^{*}$ we define

$$
\mathfrak{g}_{\alpha}=\left\{X \in \mathfrak{g}: \operatorname{ad}_{H} X=\alpha(H) X \text { for all } H \in \mathfrak{a}\right\} .
$$

If $\mathfrak{g}_{\alpha} \neq 0$, then $\alpha$ is called a root of $\mathfrak{a}$ and $m_{\alpha}:=\operatorname{dim} \mathfrak{g}_{\alpha}$ is called its multiplicity. The set of all nonzero roots is denoted by $\Delta$. If $\alpha \in \Delta$, then $-\alpha \in \Delta$. Moreover, $\sigma$ defines an isomorphism from $\mathfrak{g}_{\alpha}$ onto $\mathfrak{g}_{-\alpha}$. The connected components of $\mathfrak{a} \backslash \cup_{\alpha \in \Delta}$ ker $\alpha$ are the Weyl chambers of $\mathfrak{a}$. Pick one of them to be the positive Weyl chamber and 
denote it by $\mathfrak{a}^{+}$. A root is positive if it is positive on $\mathfrak{a}^{+}$. Let $\Delta^{+}$denote the set of positive roots. We have the following orthogonal decomposition:

$$
\mathfrak{g}=\mathfrak{g}_{0}+\sum_{\alpha \in \Delta} \mathfrak{g}_{\alpha}
$$

By definition we have $\mathfrak{a} \subset \mathfrak{g}_{0}$. In fact $\mathfrak{g}_{0}=\mathfrak{g}_{0} \cap \mathfrak{t} \oplus \mathfrak{g}_{0} \cap \mathfrak{p}$. Since $\mathfrak{a}$ is maximal, $\mathfrak{a}=\mathfrak{g}_{0} \cap \mathfrak{p}$. Moreover $\left[\mathfrak{g}_{\alpha}, \mathfrak{g}_{\beta}\right] \subset \mathfrak{g}_{\alpha+\beta}$.

It is known that for any two maximal abelian subspaces $\mathfrak{a}, \widetilde{\mathfrak{a}} \subset \mathfrak{p}$ and Weyl chambers $\mathfrak{a}^{+} \subset \mathfrak{a}, \widetilde{\mathfrak{a}}^{+} \subset \widetilde{\mathfrak{a}}$ there exists $\phi \in K$ s.t. $\phi$ maps $\mathfrak{a}$ to $\widetilde{\mathfrak{a}}$ and $\mathfrak{a}^{+}$to $\widetilde{\mathfrak{a}}^{+}$. Therefore, we may assume $\xi \in \mathfrak{a}^{+}$. Consider the linear map $T=\operatorname{ad}_{\xi}: \mathfrak{t} \rightarrow \mathfrak{p}$, and its adjoint $T^{*}=\operatorname{ad}_{\xi}: \mathfrak{p} \rightarrow \mathfrak{t}$. Indeed, for $u \in \mathfrak{t}, v \in \mathfrak{p}$

$$
\begin{aligned}
\langle T u, v\rangle & =B\left(\operatorname{ad}_{\xi} u, v\right) \\
& =-B\left(u, \operatorname{ad}_{\xi} v\right) \\
& =\left\langle u, \operatorname{ad}_{\xi} v\right\rangle .
\end{aligned}
$$

How do we calculate those eigenvalues $\lambda_{i}(\xi)$ ? Recall that they are the eigenvalues of the curvature operator $R_{\xi}: \mathfrak{p} \rightarrow \mathfrak{p}$ defined by $R_{\xi} v=-R(\xi, v) \xi$. It is a basic formula for symmetric spaces that $R_{\xi} v=-[[\xi, v], \xi]=\operatorname{ad}_{\xi} \operatorname{ad}_{\xi} v=T T^{*} v$. The curvature operator naturally extends to an endomorphism on $\mathfrak{g}=\mathfrak{t} \oplus \mathfrak{p}$, to be denoted by the same symbol $R_{\xi}$. In terms of the decomposition it is given by

$$
\left[\begin{array}{cc}
0 & T \\
T^{*} & 0
\end{array}\right]^{2}=\left[\begin{array}{cc}
T T^{*} & 0 \\
0 & T^{*} T
\end{array}\right] .
$$

Therefore

$$
\sum_{i} \sqrt{\lambda_{i}(\xi)}=\left.\operatorname{tr} \sqrt{R_{\xi}}\right|_{\mathfrak{p}}=\frac{1}{2} \operatorname{tr} \sqrt{R_{\xi}} .
$$

Then for any $v \in \mathfrak{g}$ we have decomposition

$$
v=v_{0}+\sum_{\alpha \in \Delta} v_{\alpha}
$$

Thus

$$
\begin{aligned}
R_{\xi} v & =\operatorname{ad}_{\xi} \operatorname{ad}_{\xi} v \\
& =\operatorname{ad}_{\xi}\left(\sum_{\alpha} \alpha(\xi) v_{\alpha}\right) \\
& =\sum_{\alpha} \alpha(\xi)^{2} v_{\alpha} .
\end{aligned}
$$

Therefore

$$
\begin{aligned}
\sum_{i} \sqrt{\lambda_{i}(\xi)} & =\frac{1}{2} \operatorname{tr} \sqrt{R_{\xi}} \\
& =\frac{1}{2} \sum_{\alpha \in \Delta}|\alpha(\xi)| m_{\alpha} \\
& =\sum_{\alpha \in \Delta^{+}} \alpha(\xi) m_{\alpha}
\end{aligned}
$$


Let $e_{\alpha} \in \mathfrak{a}$ be the vector s.t. $\alpha(X)=\left\langle e_{\alpha}, X\right\rangle$. Then

$$
l(\xi)=\sum_{i} \sqrt{\lambda_{i}(\xi)}=\langle\xi, H\rangle,
$$

where $H=\sum_{\alpha \in \Delta^{+}} m_{\alpha} e_{\alpha} \in \mathfrak{a}^{+}$.

Lemma 2. We have

$$
I(\widetilde{M}) \geq|H| .
$$

Proof. For each $\xi \in \mathfrak{a}^{+}$, the corresponding Busemann function $b_{\xi}$ satisfies $\Delta b_{\xi}=$ $\langle\xi, H\rangle$. Then for any open submanifold $\Omega \subset \widetilde{M}$ with compact closure and smooth boundary, integrating over $\Omega$ yields

$$
\begin{aligned}
\langle\xi, H\rangle V(\Omega) & =\int_{\Omega} \Delta b_{\xi} d v \\
& =\int_{\partial \Omega}\left\langle\nabla b_{\xi}, \nu\right\rangle d \sigma,
\end{aligned}
$$

where $\nu$ is the outer unit normal of $\partial \Omega$. Since $\left|\nabla b_{\xi}\right| \equiv 1$, we obtain

$$
\langle\xi, H\rangle V(\Omega) \leq A(\partial \Omega) \text {. }
$$

Therefore, for any $\xi \in \mathfrak{a}^{+}$,

$$
I(\widetilde{M}) \geq\langle\xi, H\rangle .
$$

Taking sup over $\xi$ yields $I(\widetilde{M}) \geq|H|$.

We claim that equality holds: $I(\widetilde{M})=|H|$. For this purpose we need to bring in another geometric invariant. Since $\widetilde{M}$ admit a compact quotient $M=\widetilde{M} / \Gamma$ by a torsion-free discrete subgroup $\Gamma \subset G$, the following limit, called the volume entropy,

$$
v=\lim _{r \rightarrow \infty} \frac{\log V(r)}{r},
$$

where $V(r)$ is the volume of the geodesic ball $B(o, r)$ with center $o$ and radius $r$, exists and is independent of the base point $o$ (cf. $\mathrm{M}]$ ). This asymptotic invariant is computed explicitly in [S] and [BCG, Appendix C]. Indeed, the volume form on $\widetilde{M}$ is given by $\prod_{i} \frac{\sinh \sqrt{\lambda_{i}(\xi)} t}{\sqrt{\lambda_{i}(\xi)}} d \sigma_{\xi} d t$ in view of (0.3). Therefore

$$
V(r)=\int_{0}^{r} \int_{\mathbb{S}^{m-1}} \prod_{i} \frac{\sinh \sqrt{\lambda_{i}(\xi)} t}{\sqrt{\lambda_{i}(\xi)}} d \sigma_{\xi} d t .
$$

From this formula it is easy to derive

$$
v=\sup _{\xi \in \mathfrak{a}^{+}} \sum_{i} \sqrt{\lambda_{i}(\xi)}=|H| .
$$

Remark 1. By a theorem of Manning $[\mathrm{M}$ the volume entropy $v$ equals the topological entropy $h$ of the geodesic flow on the unit tangent bundle $\mathcal{S} M$. In his thesis [S] Spatzier calculated the Lyapunov components for the geodesic flow. By Pesin's formula he also obtained an explicit expression for the metric entropy $h_{\mu}$, where $\mu$ is the normalized Liouville measure on $\mathcal{S} M$. Some of our calculations are essentially the same as his, but the points of view and proofs are different.

Now we can prove our main result. 
Theorem 1. Let $\widetilde{M}$ be a symmetric space of noncompact type. Then we have

$$
I=v=|H| .
$$

Proof. Given Lemma 2 and (0.5), it remains to prove $I \leq v$. This is well known. Indeed, by the definition of $I$ we have

$$
V^{\prime}(r)=A(r) \geq I V(r),
$$

where $A(r)$ is the area of the surface of the geodesic ball $B(o, r)$. Integrating gives $V(r) \geq V(1) \exp (I r)$. It follows that $v \geq I$.

As a corollary we get the following result originally proved by Karpelevič (attributed to Olshanetski [O] in [BCG]. See the discussion in [BCG, Appendix C]).

Theorem 2. Let $\widetilde{M}$ be a symmetric space of noncompact type. Then its bottom of spectrum $\lambda_{0}(\widetilde{M})$ is given by the formula

$$
\lambda_{0}(\widetilde{M})=\frac{1}{4}|H|^{2} .
$$

Proof. We recall another fundamental inequality

$$
\lambda_{0}(\widetilde{M}) \leq \frac{1}{4} v^{2}
$$

This follows easily by taking test functions $f=\exp [(v+\varepsilon) r / 2]$, with $r$ being the distance function to $o$ and $\varepsilon>0$, in (0.2) and then letting $\varepsilon \rightarrow 0$. Combining the above inequality and (0.1) we can write

$$
\frac{1}{4} I^{2} \leq \lambda_{0}(\widetilde{M}) \leq \frac{1}{4} v^{2}
$$

From this we obtain the desired identity from Theorem 1.

\section{ACKNOWLEDGMENT}

The author wishes to thank Lizhen Ji for comments and for bringing the reference $\mathrm{K}$ to the author's attention. After the paper was submitted, it was learned from a referee of related work by Ralf Spatzier in his thesis. The author thanks Ralf Spatzier for providing an electronic copy of his thesis $[\mathrm{S}]$.

\section{REFERENCES}

[BCG] G. Besson, G. Courtois, and S. Gallot, Volume et entropie minimale des espaces localement symétriques (French), Invent. Math. 103 (1991), no. 2, 417-445, DOI 10.1007/BF01239520. MR1085114 (92d:58027)

[Cha] Isaac Chavel, Riemannian geometry, 2nd ed., Cambridge Studies in Advanced Mathematics, vol. 98, Cambridge University Press, Cambridge, 2006. A modern introduction. MR2229062(2006m:53002)

[Ch] Jeff Cheeger, A lower bound for the smallest eigenvalue of the Laplacian, Problems in analysis (Papers dedicated to Salomon Bochner, 1969), Princeton Univ. Press, Princeton, N. J., 1970, pp. 195-199. MR0402831 (53 \#6645)

[E] Patrick B. Eberlein, Geometry of nonpositively curved manifolds, Chicago Lectures in Mathematics, University of Chicago Press, Chicago, IL, 1996. MR.1441541 (98h:53002)

[HI] Ernst Heintze and Hans-Christoph Im Hof, Geometry of horospheres, J. Differential Geom. 12 (1977), no. 4, 481-491 (1978). MR.512919 (80a:53051)

[H] Sigurdur Helgason, Differential geometry, Lie groups, and symmetric spaces, Pure and Applied Mathematics, vol. 80, Academic Press, Inc. [Harcourt Brace Jovanovich, Publishers], New York-London, 1978. MR514561 (80k:53081) 
[K] F. I. Karpelevič, The geometry of geodesics and the eigenfunctions of the Beltrami-Laplace operator on symmetric spaces, Trans. Moscow Math. Soc. 1965 (1967), 51-199. Amer. Math. Soc., Providence, R.I., 1967. MR0231321 (37 \#6876)

[KN] S. Kobayashi and K. Nomizu, The Foundations of Differential Geometry, Addison Wesley, Mass.

[M] Anthony Manning, Topological entropy for geodesic flows, Ann. of Math. (2) 110 (1979), no. 3, 567-573, DOI 10.2307/1971239. MR554385 (81e:58044)

[O] M. A. Olshanetski, Martin boundary of the Laplace Beltrami operator on symmetric spaces of nonpositive curvature, Math. Nauk. 1969. (Russian).

[SY] R. Schoen and S.-T. Yau, Lectures on differential geometry, Conference Proceedings and Lecture Notes in Geometry and Topology, I, International Press, Cambridge, MA, 1994. Lecture notes prepared by Wei Yue Ding, Kung Ching Chang [Gong Qing Zhang], Jia Qing Zhong and Yi Chao Xu; Translated from the Chinese by Ding and S. Y. Cheng; Preface translated from the Chinese by Kaising Tso. MR 1333601 (97d:53001)

[S] R. Spatzier, Dynamical properties of algebraic systems-a study in closed geodesics, Thesis, University of Warwick, 1983.

[Y] Shing Tung Yau, Isoperimetric constants and the first eigenvalue of a compact Riemannian manifold, Ann. Sci. École Norm. Sup. (4) 8 (1975), no. 4, 487-507. MR0397619(53 \#1478)

Department of Mathematics, Michigan State University, East Lansing, Michigan 48824

E-mail address: xwang@math.msu.edu 